\title{
The relationship of plasma Trans fatty acids with dietary inflammatory index among US adults
}

\author{
Mohsen Mazidi ${ }^{12 *}$, Hong-kai Gao ${ }^{3}$, Nitin Shivappa ${ }^{4,5,6}$, Michael D. Wirth ${ }^{4,5,6}$, James R Hebert ${ }^{4,5,6}$ \\ and Andre Pascal Kengne ${ }^{7}$
}

\begin{abstract}
Background: It has been suggested that trans fatty acids (TFAs) play an important role in cardiovascular diseases. We investigated the association between plasma TFAs and the dietary inflammatory index (DII) ${ }^{\mathrm{TM}}$ in US adults.

Methods: National Health and Nutrition Examination Survey (NHANES) participants with data on plasma TFAs measured from 1999 to 2010 were included. Energy-adjusted-DII ${ }^{\mathrm{TM}}$ (E-DII ${ }^{\mathrm{TM}}$ ) expressed per $1000 \mathrm{kcal}$ was calculated from 24-h dietary recalls. All statistical analyses accounted for the survey design and sample weights.

Results: Of the 5446 eligible participants, $46.8 \%(n=2550)$ were men. The mean age of the population was 47.1 years overall, 47.8 years for men and 46.5 years for women $(p=0.09)$. After adjustment for $C$-reactive protein, body-mass-index, smoking, race, age, education, and marital status in linear regressions, trans 9-hexadecenoic acid [ $\beta$ coefficient 0.068 (95\% Cl: 0.032 to 0.188$)$ ], trans 11 -octadecenoic acid [ $\beta$ coefficient 0.143 (95\% Cl: 0.155 to 0.310$)$ ], trans 9-octadecenoic acid [ $\beta$ coefficient 0.122 (95\% Cl: 0.120 to 0.277)], trans 9, and trans 12-octadienoic acid [ß coefficient 0.103 (95\% Cl: 0.090 to 0. 247)] were positively associated with the DII (all $p<0.001)$.
\end{abstract}

Conclusion: The association of plasma TFAs with a marker of dietary inflammation suggests an underlying mechanism in the initiation and progression of cardiovascular diseases.

Keywords: Trans fatty acids, Dietary inflammatory index, National Health and nutrition examination survey

\section{Background}

Trans-fatty acids (TFAs) are unsaturated fatty acids with at least one unsaturated, non-conjugated double bond in the trans (rather than the typical cis) configuration. Because humans do not synthesize trans-fatty acids, levels of these fatty acid isomers in serum depend on dietary intake. TFAs occur naturally in fat from ruminant animal meat, milk, and dairy fat and artificially in industrially hardened vegetable oils [1]. Dietary exposure to partially hydrogenated vegetable oils occurs through consumption of margarine and industrially processed

\footnotetext{
* Correspondence: moshen@genetics.ac.cn

${ }^{1}$ Key State Laboratory of Molecular Developmental Biology, Institute of Genetics and Developmental Biology, Chinese Academy of Sciences, Beijing 100101, China

${ }^{2}$ Institute of Genetics and Developmental Biology, International College, the University of Chinese Academy of Science, Beijing 100101, China Full list of author information is available at the end of the article
}

foods such as cakes, candies, cookies, chocolate, mayonnaise, potato chips, French fries and other deep-fat fried foods, and fast food more generally [2].

Increased dietary intake of TFAs is linked with incident cardiovascular disease (CVD), and Type 2 diabetes mellitus (T2DM) [3-6]. A recent observational study [7] and a short-term randomized trial [8] have indicated that TFAs intake increases systemic inflammation in generally healthy individuals. Because systemic inflammation is an independent risk factor for future CVD [9], these findings suggest a potential mechanism whereby TFAs may affect cardiovascular health. Recommendations from the American Heart Association have led 13 local governments to implement a TFA ban [10] and the Food and Drug Administration to require the TFAs content to be listed on the nutrition facts panel of foods and dietary supplements [10]. 
Pro- or anti-inflammatory properties have been described for a number of foods and nutrients, and extensive literature research on these food items and nutrients has led to the development of the Dietary Inflammatory Index (DII) ${ }^{\mathrm{rm}}$ [11]. The goal of this index was to use different food parameters, categorized as pro- or anti-inflammatory, in order to calculate the inflammatory property of a person's total diet $[11,12]$. Previously, the DII was associated with a range of outcomes including markers of systemic inflammation, CVD, bone mineral density, telomere length, and overall mortality [13-15]. A relationship between the DII and levels of serum inflammatory markers has been found in multiple cohorts $[11,13,16,17]$. However, no study has evaluated the association between plasma TFA concentration and DII scores. Therefore, our aim was to investigate the association between plasma TFA as an indicator of inflammation and the DII in US adults. We hypothesized that concentration of TFAs in serum will be higher with increasing DII scores.

\section{Methods \\ Population}

The National Health and Nutrition Examination Surveys (NHANES) are ongoing repeated cross sectional studies performed by the US National Center for Health Statistics (NCHS) [18]. NHANES applies a multistage probability sampling strategy, that oversamples certain subgroups of the U.S. population [19]. The NCHS Research Ethics Review Board approved the NHANES protocol and consent was obtained from all participants. Approximately 5000 subjects participate in NHANES each year, and the data are stated in 2-year cycles accessible for public domain [20]. Data collection on demographic, dietary, and behavioral information occurs through in-home administered questionnaires, though anthropometric measurements and biomarker variables are collected by trained subjects applying the mobile exam clinics (MEC). The interview consists of questions on socio-demographic characteristics (age, gender, education and race/Hispanic origin). More comprehensive information on the NHANES protocol is accessible elsewhere [21]. This study was based on analysis of data from the 1999 to 2010 NHANES cycles. Analyses were limited to subjects aged 18 years and older.

\section{Plasma trans fatty acids}

This measurement procedure determines the total (free and esterified) content of selected TFAs in plasma and provides results in concentration units as well as percent units (trans fatty acids as percent of total fatty acids). The fatty acids in plasma are converted into free fatty acids by subsequent acidic and alkaline hydrolysis. The free fatty acids are extracted from the sample solution using liquid-liquid extraction and derivatized with pentafluorobenzylbromide (PFB-Br). The derivatized fatty acids are separated by capillary gas chromatography and detected by mass spectrometry using negative chemical ionization. The fatty acids are identified based on their chromatographic retention time and on the specific mass to charge ratio of the ion formed in the ion source. Retention times are compared against those obtained with known standards. Quantitation is performed with standard solution using stable isotope-labelled fatty acids as internal standards. To calculate trans fatty acids as percent of total fatty acids, 29 fatty acids are determined with this measurement procedure. These fatty acids cover over $95 \%$ of all fatty acids reported in plasma. This method determines the following four trans fatty acids: trans-9-hexadecenoic acid (palmitelaidic acid, C16:1n7 t), trans-9-octadecenoic acid (elaidic acid, C18:1n-9 t), trans-11-octadecenoic acid (vaccenic acid, C18:1n-7 t-), trans-9, trans-12-octadecadienoic acid (linolelaidic acid, C18:2n-6 t, 9 t) [22].

\section{Diet and dietary inflammatory index}

The development and validation of the DII have been described in detail elsewhere [12]. In short, nearly 2000 research articles, published between 1950 and 2010, examining the relationship between components of diet and pro-inflammatory cytokines (i.e., IL-1 $\beta$, IL-6, tumour necrosis factor [TNF]- $\alpha$, and C-reactive protein (CRP)) and anti-inflammatory cytokines (IL-4 and IL-10) were reviewed. This search revealed that 45 different food parameters (mostly micro, macro nutrients and flavanoids plus some individual food items) had a sufficiently robust literature to allow for scoring. Articles showing a positive association between the food parameters and the pro-inflammatory cytokines or a negative association with anti-inflammatory cytokines received a value of +1 . If the food parameters were associated with reduced pro-inflammatory or increased antiinflammatory cytokines, the article received a value of -1 . Null values were set to 0 . These scores were weighted based on study design. For example, randomized control trials received the greatest weight and cell culture the lowest weight. These scores and the weights were used to create pro- and anti-inflammatory fractions for each food parameter. The anti-inflammatory fraction was subtracted from the pro-inflammatory fraction to create the "article effect score" for each of the 45 food parameters. Additionally, DII calculation is linked to a regionally representative world database. The world database contains standard means and deviations for the 45 food parameters from 11 populations around the world (i.e., United States, United Kingdom, Bahrain, Mexico, Australia, South Korea, Taiwan, India, New Zealand, Japan, and Denmark) [12]. Dietary data in 
NHANES were collected using 24-h dietary recall interviews (24HR) conducted at the MEC [23]. The dietary interviews were administered by trained staff and the USDA's Food Surveys Research Group was responsible for the dietary data collection methodology, maintenance of the databases used to code and process the data, and data review and processing [24]. 24HR-derived dietary information was used to calculate DII scores for all subjects, as described in detail elsewhere [12]. Higher (i.e., more positive) scores tend to indicate more proinflammatory diets and more negative values are more anti-inflammatory [12]. To control for the effect of total energy intake, the DII was calculated per $1000 \mathrm{cal}$ of food consumed, which requires using the energystandardized version of the world database.

\section{Statistical analysis}

We conducted the analyses according to the Center for Diseases Control and Prevention guidelines for analysis of complex NHANES data, accounting for the masked variance and using the proposed weighting methodology [25-28]. We computed C-reactive protein, body-mass-index, smoking, race, age, education, and marital status-adjusted plasma TFAs level across quartiles of the DII by using analysis of covariance (ANCOVA) with Bonferroni correction. To determine any association between plasma TFAs and DII, we used multivariable-adjusted linear regression models adjusted for C-reactive protein, body-massindex, smoking, race, age, education, marital status. All tests were two sided, and the level of significance as set at $p<0.05$. We have applied complex sample analysis to deal with unequal probabilities of selection, nonresponse bias, and oversampling.

\section{Results}

Of the 11,973 eligible participants, $47.8 \%(n=5725)$ were men. The mean age was 47.1 years overall, 47.8 years in men and 46.5 years in women $(p=0.085)$. In all, $40.6 \%(n=4864)$ of the participants had completed more than high school, $21.1 \%(n=2522)$ had completed high school, while $30.6 \%(n=3672)$ had completed less than high school. White (non-Hispanic) represented $45.1 \%(n=5405)$ of the participants, Blacks $18.6 \%(n=2230)$ and Mexican-Americans $23.2 \%$ $(n=2779)$. The distribution of marital status was $50.9 \%$ (5659) for married, $9.5 \%(n=1055)$ for widowed and 9.3\% $(n=1035)$ for divorced. Means and standard deviations for TFAs for subjects with information on TFAs concentration were $1.55 \pm 0.52 \mu \mathrm{mol} / \mathrm{L}$ for trans 9-hexadecenoic acid, $3.18 \pm 0.61 \mu \mathrm{mol} / \mathrm{L}$ for trans 11 octadecenoic acid, $2.96 \pm 0.67 \mu \mathrm{mol} / \mathrm{L}$ for trans 9octadecenoic acid and $0.68 \pm 0.21 \mu \mathrm{mol} / \mathrm{L}$ for trans 9 , trans 12-octadienoic acid, respectively. Mean BMI was $28.5 \pm 6.6 \mathrm{Kg} / \mathrm{m}^{2}$ overall, $28.0 \pm 5.7 \mathrm{Kg} / \mathrm{m}^{2}$ in men and $29.0 \pm 7.3 \mathrm{Kg} / \mathrm{m}^{2}$ in women $(p=0.012)$. DII score ranged from -5.33 to 4.24 with a median of -0.01 (25th-75th percentiles: $-1.40-1.20$ ).

Adjusted mean Trans 9-hexadecenoic acid, trans 11octadecenoic acid, trans 9-octadecenoic acid, trans 9, and trans 12-octadienoic acid significantly increased across increasing quarters of the DII $(p<0.001$, Table 1$)$. In $C$-reactive protein, body-mass-index, smoking, race, age, education, and marital status adjusted linear regressions, Trans 9-hexadecenoic acid [ $\beta$ coefficient $(\beta) 0.068$ (95\% CI: 0.032 to 0.188$)$ ], trans 11 -octadecenoic acid [ $\beta$ 0.143 (95\% CI: 0.155 to 0.310 )], trans 9-octadecenoic acid [ $\beta 0.122$ (95\% CI: 0.120 to 0.277 )], and trans 9, trans 12-octadienoic acid [ $\beta 0.103$ (95\% CI: 0.090 to 0.247 )] were directly related to DII score (all $p<0.001$ ).

Table 1 adjusted (C-reactive protein, body mass-index, smoking, race, age, education, marital status)mean of trans fatty acids across the quarters of the DII

\begin{tabular}{|c|c|c|c|c|c|c|}
\hline \multicolumn{2}{|l|}{ Variables } & \multicolumn{4}{|c|}{ Quarters of the dietary inflammatory index } & \multirow[t]{3}{*}{$P$-value } \\
\hline & & \multirow{2}{*}{$\begin{array}{l}\text { Quarter } 1 \\
-5.33 \text { to }-1.40\end{array}$} & \multirow{2}{*}{$\begin{array}{l}\text { Quarter } 2 \\
-1.40 \text { to }-0.02\end{array}$} & \multirow{2}{*}{$\begin{array}{l}\text { Quarter } 3 \\
-0.02 \text { to } 1.20\end{array}$} & \multirow{2}{*}{$\begin{array}{l}\text { Quarter } 4 \\
1.20 \text { to } 4.24\end{array}$} & \\
\hline \multicolumn{2}{|c|}{ Min and Max of DII } & & & & & \\
\hline \multirow[t]{4}{*}{ crude } & trans 9-hexadecenoic acid & $1.57 \pm 0.03$ & $1.63 \pm 0.02$ & $1.64 \pm 0.03$ & $1.56 \pm 0.02$ & 0.095 \\
\hline & trans 11 -octadecenoic acid & $3.10 \pm 0.02$ & $3.20 \pm 0.02$ & $3.29 \pm 0.05$ & $3.32 \pm 0.07$ & $<0.001$ \\
\hline & trans 9-octadecenoic acid & $2.92 \pm 0.04$ & $2.95 \pm 0.02$ & $3.02 \pm 0.05$ & $3.12 \pm 0.03$ & $<0.001$ \\
\hline & trans 9, trans 12-octadienoic acid & $0.65 \pm 0.04$ & $0.74 \pm 0.02$ & $0.76 \pm 0.03$ & $0.82 \pm 0.02$ & $<0.001$ \\
\hline \multirow[t]{4}{*}{ adjusted } & trans 9-hexadecenoic acid & $1.37 \pm 0.04$ & $1.40 \pm 0.04$ & $1.44 \pm 0.02$ & $1.50 \pm 0.04$ & $<0.001$ \\
\hline & trans 11 -octadecenoic acid & $2.91 \pm 0.03$ & $2.96 \pm 0.05$ & $3.06 \pm 0.04$ & $3.32 \pm 0.05$ & $<0.001$ \\
\hline & trans 9-octadecenoic acid & $2.80 \pm 0.04$ & $2.82 \pm 0.02$ & $2.87 \pm 0.03$ & $2.96 \pm 0.04$ & $<0.001$ \\
\hline & trans 9, trans 12 -octadienoic acid & $0.62 \pm 0.04$ & $0.69 \pm 0.04$ & $0.72 \pm 0.03$ & $0.80 \pm 0.03$ & $<0.001$ \\
\hline
\end{tabular}




\section{Discussion}

In this population-based sample, we have evaluated the association between plasma TFA and the DII. We found a positive association between plasma TFA and DII scores, which remained significant even after adjusting for a range the potential confounding factors.

Evidence from both observational and experimental studies indicates that TFAs are pro-inflammatory $[5,7$, $29,30]$, which is in line with our findings. The mechanisms underlying these effects are not well-established, but may involve TFAs incorporation into endothelial cell, monocyte/macrophage, or adipocyte cell membranes (affecting membrane signalling pathway relating to inflammation) or ligand-dependent effects on peroxisome-proliferator-activated receptor-g (PPAR-g) or retinoid X receptor (RXR) pathways [5, 7]. Activation of inflammatory responses and endothelial dysfunction may represent important mediating pathways between TFAs consumption and the risk of coronary heart diseases, sudden death, and T2DM [29]. It has been reported that TFA treatment of human aortic endothelial cell (HAEC) significantly increased the expression of endothelial adhesion molecules, including intercellular adhesion molecule-1 (CD54) and vitronectin receptor (CD51/CD61) [31]. Incorporation of TFA into membranes increased HAEC adhesion to fibronectin- or vitronectin-coated plates by 1.5 - to 2 -fold, respectively [31]. Neutrophil and monocyte adhesion to HAEC monolayers was nearly proportional to adhesion molecule expression. TFA treatment also induced the release of monocyte chemoattractant protein-1 by nearly threefold in non-stimulated HAEC [31]. TFA elevates inflammatory markers such as CRP, TNF alpha, and IL-6 $[8,32]$. Mozaffarian et al. observed that TFA levels were positively associated with interleukin (IL) $1 ß$, IL-1 receptor antagonist, IL-10, tumor necrosis factor (TNF) $\alpha$, TNF receptor 2 , monocyte chemoattractant protein 1 , and brain natriuretic peptide [7].

Some of the deleterious actions of the TFAs such as increasing insulin resistance have been attributed to its pro-inflammatory action. A recent study in mice demonstrated increased insulin resistance and signs of local inflammation in adipose tissue, as well as induction of cytokine gene expression after treatment with trans10cis12-CLA [33]. Adipocytes exposed to CLA secreted more pro-inflammatory cytokines than controls, and there was an increased macrophage infiltration in adipose tissue treated with trans10cis12-CLA [33]. Another mechanism, as indicated by many studies in CLAfed mice, is down-regulation of PPAR- $\gamma$ in adipose tissue [33-35], resulting in subsequent peripheral insulin resistance in skeletal muscle. Data in mice also suggest that CLA could promote a lipodystrophic state, with redistribution of fat stores, i.e. increased liver fat accumulation and decreased peripheral fat, also consistent with down-regulation of PPAR-g [35].

The effects of TFA consumption on cardiovascular events have not been evaluated in randomized controlled trials in humans. Such trials are unlikely to be performed, given the cost considerations, practicality, compliance and, perhaps most importantly, the ethical limitations of randomizing individuals to an intervention with strong evidence for significant harm. A prior metaanalysis of observational studies reported pooled relative risk estimates for CHD of 1.22 (95\% confidence interval 1.08 to 1.38 ) for highest level of total intake of TFAs; 1.30 ( 0.80 to 2.14 ) for intake of industrially produced TFAs; and 0.93 (0.74 to 1.18$)$ for intake of ruminant derived TFAs [36]. This suggests that industrially produced TFAs might increase the risk of CHD, though this also could reflect the low levels of ruminant derived TFAs compared with the higher doses of industrially produced TFAs typically consumed in studies and availability in the food supply [37]. A meta-analysis by Souza et al., reported that a $2 \%$ increase in energy intake from TFAs was associated with a $25 \%$ increased risk of CHD and $31 \%$ increase in CHD mortality [38]. Other metaanalyses also have reached the same conclusion $[5,39]$.

With regard to stroke, at least two prospective studies assessed the association between TFAs and ischemic stroke, and yielded inconsistent results [40, 41]. One study in men showed no association with stroke [40]; the other, in women, showed a positive association in those who did not take aspirin [41]. Furthermore, the association with TFAs was significant only for lacunar stroke, with a trend for haemorrhagic stroke, but not for stroke of cardioembolic origin. A nested case-control study conducted within the Women's Health Initiative Observational Study with 10 year follow-up found no association between serum TFAs 16:1, 18:1, or 18:2 and ischemic stroke [42].

This study has several strengths. To our knowledge, it is the largest study of the association of plasma TFAs with the DII. The study is sufficiently powered to test the targeted associations. Participants were a random sample of the general population and therefore the results obtained from nationally representative samples can be extrapolated to the general population. As the data collection was performed on all days of the week throughout the year in NHANES, the potential for selection bias is very low [43, 44]. The findings from our study have to be considered in the context of some study limitations. The crosssectional nature does not allow inference about causality; it is possible there is reverse causation, whereas it is not a case for our results since increased plasma TFAs cannot cause someone to eat a pro-inflammatory diet. 


\section{Conclusions}

Understanding the interplay between plasma TFA and DII is a necessary and important step toward any application of the resulting knowledge for public health policy and action. Our findings provide evidence on the association between plasma TFAs with the DII. The association of plasma TFAs and with a marker of inflammation suggests an underlying mechanism of harmful health effects.

\begin{abstract}
Abbreviations
ANCOVA: Analysis of covariance; CRP: C-reactive protein; CVD: Cardiovascular disease; DII: Dietary inflammatory index; E-DII: Energy-adjusted-dietary inflammatory index; HAEC: Human aortic endothelial cell; MEC: Mobile exam clinics; NCHS: National Center for Health Statistics; NHANES: National Health and Nutrition Examination Survey; PFB-Br: Pentafluorobenzylbromide; PPAR-g: Peroxisome-proliferator-activated receptor-g; RXR: Retinoid X receptor; T2DM: type 2 diabetes mellitus; TFA: Trans fatty acids; TNF: Tumour necrosis factor
\end{abstract}

\section{Acknowledgments}

MM was supported by a TWAS and Chinese Academy of Sciences. Drs. Shivappa, Wirth, and Hébert were supported by grant number R44DK103377 to $\mathrm{CH}$ from the United States National Institute of Diabetes and Digestive and Kidney Diseases.

\section{Funding}

None.

\section{Availability of data and materials}

All the data are from public access database.

\section{Authors' contributions}

MM, contributed to the study concept and design, data analysis and interpretation, and drafting of the manuscript. NS, MDW, JRH, data analyses and critical revision of the manuscript for important intellectual content. APK, contributed to the design of the study, data analyses and critical revision of the manuscript for important intellectual content, and drafting of the manuscript.

\section{Ethics approval and consent to participate}

For the data collection and physical examination of the NHANES, informed consent was obtained from all adult participants, and the National Centre for Health Statistics Research Ethics Review Board approved the protocol.

\section{Consent for publication}

For the data collection and physical examination of the NHANES, informed consent (publication) was obtained from all adult participants, and the National Centre for Health Statistics Research Ethics Review Board approved the protocol.

\section{Competing interests}

Dr. James R. Hébert owns controlling interest in Connecting Health Innovations LLC (CHI), a company planning to license the right to his invention of the dietary inflammatory index (DII) from the University of South Carolina in order to develop computer and smart phone applications for patient counseling and dietary intervention in clinical settings. Drs. Nitin Shivappa and Michael Wirth are employees of $\mathrm{CHI}$. All other co-authors have nothing to declare.

\section{Author details}

${ }^{1}$ Key State Laboratory of Molecular Developmental Biology, Institute of Genetics and Developmental Biology, Chinese Academy of Sciences, Beijing 100101, China. ${ }^{2}$ Institute of Genetics and Developmental Biology, International College, the University of Chinese Academy of Science, Beijing 100101, China. ${ }^{3}$ The General Hospital of Chinese People's Armed Police Forces, Beijing, China. ${ }^{4}$ Cancer Prevention and Control Program, University of South Carolina, 915 Greene Street, Suite 200, Columbia, SC 29208, USA. ${ }^{5}$ Department of Epidemiology and Biostatistics, University of South Carolina,
915 Greene Street, Suite 400, Columbia, SC 29208, USA. ${ }^{6}$ Connecting Health Innovations, LLC, 1417 Gregg Street, Columbia, SC 29201, USA.

${ }^{7}$ Non-Communicable Diseases Research Unit, South African Medical Research Council and University of Cape Town, Cape Town, South Africa.

Received: 4 April 2017 Accepted: 22 June 2017

Published online: 04 August 2017

\section{References}

1. Sommerfeld M. Trans unsaturated fatty acids in natural products and processed foods. Prog Lipid Res. 1983;22(3):221-33.

2. Chajes V, Thiebaut AC, Rotival M, Gauthier E, Maillard V, Boutron-Ruault MC, et al. Association between serum trans-monounsaturated fatty acids and breast cancer risk in the E3N-EPIC study. Am J Epidemiol. 2008; 167(11):1312-20.

3. Stender $S$, Dyerberg J. Influence of trans fatty acids on health. Ann Nutr Metab. 2004;48(2):61-6.

4. Zhao X, Shen C, Zhu H, Wang C, Liu X, Sun X, et al. Trans-Fatty Acids Aggravate Obesity, Insulin Resistance and Hepatic Steatosis in C57BL/6 Mice, Possibly by Suppressing the IRS1 Dependent Pathway. Molecules (Basel, Switzerland). 2016;21(6).

5. Mozaffarian D, Aro A, Willett WC. Health effects of trans-fatty acids: experimental and observational evidence. Eur J Clin Nutr. 2009; 63(Suppl 2):S5-21.

6. Barnard ND, Bunner AE, Agarwal U. Saturated and trans fats and dementia: a systematic review. Neurobiol Aging. 2014:35(Suppl 2):S65-73.

7. Mozaffarian D, Pischon T, Hankinson SE, Rifai N, Joshipura K, Willett WC, et al. Dietary intake of trans fatty acids and systemic inflammation in women. Am J Clin Nutr. 2004;79(4):606-12.

8. Baer DJ, Judd JT, Clevidence BA, Tracy RP. Dietary fatty acids affect plasma markers of inflammation in healthy men fed controlled diets: a randomized crossover study. Am J Clin Nutr. 2004;79(6):969-73.

9. Libby P, Ridker PM, Maseri A. Inflammation and atherosclerosis. Circulation. 2002;105(9):1135-43

10. Aronis KN, Khan SM, Mantzoros CS. Effects of trans fatty acids on glucose homeostasis: a meta-analysis of randomized, placebo-controlled clinical trials. Am J Clin Nutr. 2012:96(5):1093-9.

11. Cavicchia PP, Steck SE, Hurley TG, Hussey JR, Ma Y, Ockene IS, et al. A new dietary inflammatory index predicts interval changes in serum highsensitivity C-reactive protein. J Nutr. 2009;139(12):2365-72.

12. Shivappa N, Steck SE, Hurley TG, Hussey JR, Hebert JR. Designing and developing a literature-derived, population-based dietary inflammatory index. Public Health Nutr. 2014;17(8):1689-96.

13. Wirth MD, Burch J, Shivappa N, Violanti JM, Burchfiel CM, Fekedulegn D, et al. Association of a dietary inflammatory index with inflammatory indices and metabolic syndrome among police officers. J Occup Environ Med. 2014;56(9):986-9.

14. Garcia-Arellano A, Ramallal R, Ruiz-Canela M, Salas-Salvado J, Corella D, Shivappa N, et al. Dietary inflammatory index and incidence of cardiovascular disease in the PREDIMED study. Nutrients. 2015:7(6):4124-38.

15. Garcia-Calzon S, Zalba G, Ruiz-Canela M, Shivappa N, Hebert JR, Martinez JA, et al. Dietary inflammatory index and telomere length in subjects with a high cardiovascular disease risk from the PREDIMED-NAVARRA study: cross-sectional and longitudinal analyses over 5 y. Am J Clin Nutr. 2015;102(4):897-904.

16. Shivappa N, Steck SE, Hurley TG, Hussey JR, Ma Y, Ockene IS, et al. A population-based dietary inflammatory index predicts levels of C-reactive protein in the seasonal variation of blood cholesterol study (SEASONS). Public Health Nutr. 2014;17(8):1825-33.

17. Shivappa N, Hebert JR, Rietzschel ER, De Buyzere ML, Langlois M, Debruyne E, et al. Associations between dietary inflammatory index and inflammatory markers in the Asklepios study. Br J Nutr. 2015;113(4):665-71.

18. Mazidi M, Kengne AP, Sahebkar A, Banach M. Telomere Length Is Associated With Cardiometabolic Factors in US Adults. Angiology. 2017:3319717712860. doi:10.1177/0003319717712860. [Epub ahead of print].

19. Mazidi M, Penson P, Banach M. Association between telomere length and complete blood count in US adults. Arch Med Sci. 2017:13(3):601-5. doi:10. 5114/aoms.2017.67281. Epub 2017 Apr 20

20. Mazidi M, Kengne AP, P Mikhailidis D, F Cicero A, Banach M. Effects of selected dietary constituents on high-sensitivity C-reactive protein levels in U.S. adults. Ann Med. 2017:1-6. doi:10.1080/07853890.2017.1325967. [Epub ahead of print]. 
21. Kalk WJ, Joffe BI. The metabolic syndrome, insulin resistance, and its surrogates in African and white subjects with type 2 diabetes in South Africa. Metab Syndr Relat Disord. 2008;6(4):247-55.

22. Teegala SM, Willett WC, Mozaffarian D. Consumption and health effects of trans fatty acids: a review. J AOAC Int. 2009;92(5):1250-7.

23. Mazidi M, Pennathur S, Afshinnia F. Link of dietary patterns with metabolic syndrome: analysis of the National Health and Nutrition Examination Survey. Nutr Diabetes. 2017;7(3):e255. doi:10.1038/nutd.2017.11.

24. Mazidi M, Kengne AP. Nutrient patterns and their relationship with general and central obesity in US adults. Eur J Clin Invest. 2017. doi:10.1111/eci. 12745. [Epub ahead of print].

25. Statistics. NCfH. Analytic and reporting guidelines wwwn.cdc.gov/nchs/nhanes/ continuousnhanes nhanes 03 04/nhanes analytic guidelines dec 2005.pdf.

26. Mazidi M, Gao HK, Vatanparast H, Kengne AP. Impact of the dietary fatty acid intake on C-reactive protein levels in US adults. Medicine (Baltimore). 2017;96(7):e5736. doi:10.1097/MD.0000000000005736.

27. Mazidi M, Kengne AP, Banach M. Mineral and vitamin consumption and telomere length among adults in the United States. Pol Arch Intern Med. 2017;127(2):87-90. doi:10.20452/pamw.3927. Epub 2017 Feb 2

28. Mazidi M, Michos ED, Banach $M$. The association of telomere length and serum 25-hydroxyvitamin D levels in US adults: the National Health and Nutrition Examination Survey. Arch Med Sci. 2017;13(1):61-5. doi:10.5114/aoms.2017.64714. Epub 2016 Dec 19.

29. Mozaffarian D, Katan MB, Ascherio A, Stampfer MJ, Willett WC. Trans fatty acids and cardiovascular disease. N Engl J Med. 2006:354(15):1601-13.

30. Mozaffarian D, Rimm EB, King IB, Lawler RL, McDonald GB, Levy WC. Trans fatty acids and systemic inflammation in heart failure. Am J Clin Nutr. 2004;80(6):1521-5.

31. Harvey KA, Walker $\mathrm{CL}, \mathrm{Xu} Z$, Whitley P, Siddiqui RA. Trans fatty acids: induction of a pro-inflammatory phenotype in endothelial cells. Lipids. 2012;47(7):647-57.

32. Han SN, Leka LS, Lichtenstein AH, Ausman LM, Schaefer EJ, Meydani SN. Effect of hydrogenated and saturated, relative to polyunsaturated, fat on immune and inflammatory responses of adults with moderate hypercholesterolemia. J Lipid Res. 2002;43(3):445-52.

33. Poirier H, Shapiro JS, Kim RJ, Lazar MA. Nutritional supplementation with trans-10, cis-12-conjugated linoleic acid induces inflammation of white adipose tissue. Diabetes. 2006;55(6):1634-41.

34. Tsuboyama-Kasaoka N, Takahashi M, Tanemura K, Kim HJ, Tange T, Okuyama $\mathrm{H}$, et al. Conjugated linoleic acid supplementation reduces adipose tissue by apoptosis and develops lipodystrophy in mice. Diabetes. 2000;49(9):1534-42.

35. Granlund L, Juvet LK, Pedersen Jl, Nebb HI. Trans10, cis12-conjugated linoleic acid prevents triacylglycerol accumulation in adipocytes by acting as a PPARgamma modulator. J Lipid Res. 2003;44(8):1441-52.

36. Bendsen NT, Christensen R, Bartels EM, Astrup A. Consumption of industrial and ruminant trans fatty acids and risk of coronary heart disease: a systematic review and meta-analysis of cohort studies. Eur J Clin Nutr. 2011;65(7):773-83

37. Brouwer IA, Wanders AJ, Katan MB. Effect of animal and industrial trans fatty acids on HDL and LDL cholesterol levels in humans-a quantitative review. PLoS One. 2010;5(3):e9434.

38. de Souza RJ, Mente A, Maroleanu A, Cozma Al, Ha V, Kishibe T, et al. Intake of saturated and trans unsaturated fatty acids and risk of all cause mortality, cardiovascular disease, and type 2 diabetes: systematic review and meta-analysis of observational studies. BMJ. 2015;351:h3978.

39. Skeaff CM, Miller J. Dietary fat and coronary heart disease: summary of evidence from prospective cohort and randomised controlled trials. Ann Nutr Metab. 2009:55(1-3):173-201.

40. He K, Merchant A, Rimm EB, Rosner BA, Stampfer MJ, Willett WC, et al. Dietary fat intake and risk of stroke in male US healthcare professionals: 14 year prospective cohort study. BMJ. 2003;327(7418):777-82

41. Yaemsiri S, Sen S, Tinker L, Rosamond W, Wassertheil-Smoller S, He K. Trans fat, aspirin, and ischemic stroke in postmenopausal women. Ann Neurol. 2012;72(5):704-15.

42. Wang Q, Imamura F, Lemaitre RN, Rimm EB, Wang M, King IB, et al. Plasma phospholipid trans-fatty acids levels, cardiovascular diseases, and total mortality: the cardiovascular health study. J Am Heart Assoc. 2014;3(4).
43. Tooze JA, Midthune D, Dodd KW, Freedman LS, Krebs-Smith SM, Subar AF, et al. A new statistical method for estimating the usual intake of episodically consumed foods with application to their distribution. J Am Diet Assoc. 2006;106(10):1575-87.

44. Guenther PM, Ding EL, Rimm EB. Alcoholic beverage consumption by adults compared to dietary guidelines: results of the National Health and nutrition examination survey, 2009-2010. J Acad Nutr Diet. 2013;113(4):546-50.

\section{Submit your next manuscript to BioMed Central and we will help you at every step:}

- We accept pre-submission inquiries

- Our selector tool helps you to find the most relevant journal

- We provide round the clock customer support

- Convenient online submission

- Thorough peer review

- Inclusion in PubMed and all major indexing services

- Maximum visibility for your research

Submit your manuscript at www.biomedcentral.com/submit
Biomed Central 